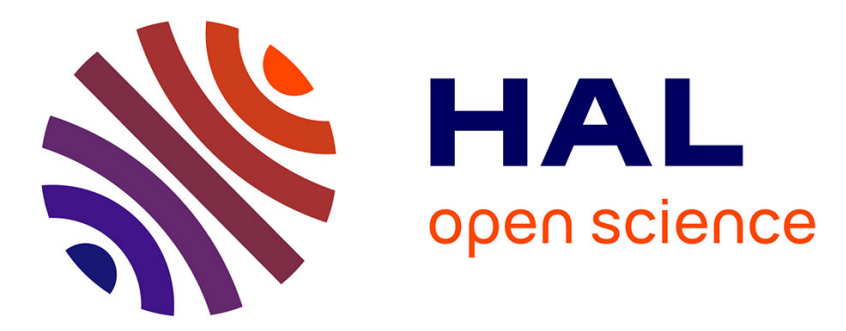

\title{
Delay-dependent stability analysis of linear systems with time-varying delay
}

Yassine Ariba, Frédéric Gouaisbaut

\section{To cite this version:}

Yassine Ariba, Frédéric Gouaisbaut. Delay-dependent stability analysis of linear systems with timevarying delay. 46th IEEE Conference on Decision and Control, Dec 2007, New Orleans, United States. pp.2053 - 2058, 10.1109/CDC.2007.4434619 . hal-00357911

\section{HAL Id: hal-00357911 \\ https://hal.science/hal-00357911}

Submitted on 2 Feb 2009

HAL is a multi-disciplinary open access archive for the deposit and dissemination of scientific research documents, whether they are published or not. The documents may come from teaching and research institutions in France or abroad, or from public or private research centers.
L'archive ouverte pluridisciplinaire HAL, est destinée au dépôt et à la diffusion de documents scientifiques de niveau recherche, publiés ou non, émanant des établissements d'enseignement et de recherche français ou étrangers, des laboratoires publics ou privés. 


\title{
Delay-dependent stability analysis of linear systems with time-varying delay
}

\author{
Yassine Ariba*† and Frédéric Gouaisbaut**
}

December 2007

\begin{abstract}
Stability analysis of linear systems with time-varying delay is investigated. In order to highlight the relations between the variation of the delay and the states, redundant equations are introduced to construct a new modeling of the delay system. New types of Lyapunov Krasovskii functionals are then proposed allowing to reduce the conservatism of the stability criterion. Delay dependent stability conditions are then formulated in terms of linear matrix inequalities (LMI). Finally, an example shows the effectiveness of the proposed methodology.
\end{abstract}

\section{INTRODUCTION}

During the last decades, stability of linear time delay systems have attracted a lot of attention [5], [13], [14], [17], [9] and references therein. Numerous tools for estimating the stability of linear time delay systems have been successfully exploited. The first classical technique relies on the study of the roots of the associated characteristic equation, a quasipolynomial in $s$ and $e^{-h s}$. Even very effective in practice [18], these approaches reveal themselves quite complicated when uncommensurate delays, robustness issues or time varying delays are considered. The stability of time-delay systems can be also studied in an Input-Output framework [5], [12], [8, [7] and [9]. In this case, methods aim at embed the delay as an uncertain operator and hence transform the original delay system into a linear system submitted to a perturbation. Then, the use of classical robustness tools like Small Gain theorem, IQC or Quadratic Separation approach allow then to develop effective criteria [22], 21], [12], [16] and [7]. In this framework, the source of induced conservatism is clear and generally comes from the choice of the interconnection (often related to the choice of a model transformation) and the choice of the uncertainty set which covers the delay operator.

Another very popular approach relies on the use of a Lyapunov-Krasovskii functional. Indeed, for a linear time delay system, some general functional can be found [9] but is very difficult to handle. That is the reason why more simple and thus more conservative Lyapunov-Krasovskii functional have been

\footnotetext{
*Université de Toulouse; UPS, 118 Route de Narbonne, F-31062 Toulouse, France.

${ }^{\dagger}$ LAAS; CNRS; 7, avenue du Colonel Roche, F-31077 Toulouse, France. Email: yariba@laas.fr, fgouaisb@laas.fr
} 
proposed. Generally, all these approach have to tackle with two main difficulties. The first one is the choice of the model transformation. The second problem lies on the bound of some cross terms which appear in the derivative of the Lyapunov functional. The present paper brings a contribution to the first issue using an augmented model of time-varying delay systems. This method is closely related to the technique proposed by [3] in a robustness framework. In this latter paper, an extended state variable is constructed to deal with the stability of a linear uncertain system. This modelling allows then to develop a parameter-dependent Lyapunov function. For time delay systems, it was shown that introducing redundant differential equations shifted in time allows to build conditions that improve results (see [1] for independent of delay criteria and [6] for delay dependent criteria). In [11], an augmented Lyapunov functional is proposed and is based on the use of the state variable and its derivative and shows interesting results especially for robustness issues. In this paper, using the derivative operator, a different method is proposed to consider augmented time-varying delay systems and then to provide new delay dependent stability criteria.

The paper is organized as follows. In section 2, a first result is derived from a Lyapunov-Krasovskii functional developed in 10] for delay dependent stability analysis. This section aims at exhibiting another formulation of the analysis problem for time-varying delay systems. Then, in section 3 we expose the two main results of this paper: the use of the system derivative and an additional term for the Lyapunov-Krasovskii functional. Finally, the following section 4 is devoted to a numerical experiment that illustrate the proposed approach.

Notations: For two symmetric matrices, $A$ and $B, A>(\geq) B$ means that $A-B$ is (semi-) positive definite. $A^{T}$ denotes the transpose of $A$. $1_{\mathrm{n}}$ and $0_{\mathrm{m} \times \mathrm{n}}$ denote respectively the identity matrix of size $n$ and null matrix of size $m \times n$. If the context allows it, the dimensions of these matrices are often omitted. For a given matrix $B \in \mathrm{R}^{\mathrm{m} \times \mathrm{n}}$ such that $\operatorname{rank}(B)=r$, we define $B^{\perp} \in \mathrm{R}^{\mathrm{n} \times(\mathrm{n}-\mathrm{r})}$ the right orthogonal complement of $B$ by $B B^{\perp}=0$.

\section{A first result on stability}

Consider the following linear time delay system:

$$
\begin{cases}\dot{x}(t)=A x(t)+A_{d} x(t-h(t)), & \forall t \geq 0, \\ x(t)=\phi(t), & \forall t \in\left[-h_{m}, 0\right],\end{cases}
$$

where $x(t) \in \mathrm{R}^{\mathrm{n}}$ is the state vector, $A, A_{d} \in \mathrm{R}^{\mathrm{n} \times \mathrm{n}}$ are known constant matrices and $\phi$ is the initial condition. The delay, $h(t)$, is assumed to be a time-varying continuous function that satisfies

$$
0 \leq h(t) \leq h_{m}
$$

where $h_{m}>0$ may be arbitrarily large if delay independent conditions are looked for. Furthermore, we also assume that a bound on the derivative of $\dot{h}(t)$ is provided :

$$
|\dot{h}(t)| \leq d
$$

The aim of this section is to derive some conditions on $h_{m}$, the upperbound which ensure the stability of (11) for a given value $d$ by using a Lyapunov- 
Krasovskii framework. The next theorem gives the following delay dependent result for system (1).

Theorem 1 Given scalars $h_{m}>0$ and $d \geq 0$, system (1) is asymptotically stable for any time-varying delay $h(t)$ satisfying (国) and (3) if there exists $n \times n$ matrices $P>0, Q_{i}>0, i=\{1,2\}$ and $R>0$ such that the following $L M I$ holds:

$$
S^{\perp^{T}} \Gamma S^{\perp}<0
$$

where

$$
\begin{gathered}
S=\left[\begin{array}{lccc}
-1 & A & A_{d} & 0
\end{array}\right] \text { and } \\
\Gamma=\left[\begin{array}{cccc}
h_{m} \mathbf{R} & \mathbf{P} & 0 & 0 \\
\mathbf{P} & T & \frac{1}{h_{m}} \mathbf{R} & 0 \\
0 & \frac{1}{h_{m}} \mathbf{R} & U & \frac{1}{h_{m}} \mathbf{R} \\
0 & 0 & \frac{1}{h_{m}} \mathbf{R} & V
\end{array}\right] .
\end{gathered}
$$

with

$$
\begin{aligned}
T & =\mathbf{Q}_{\mathbf{1}}+\mathbf{Q}_{\mathbf{2}}-\frac{1}{h_{m}} \mathbf{R}, \\
U & =-(1-d) \mathbf{Q}_{\mathbf{1}}-\frac{2}{h_{m}} \mathbf{R}, \\
V & =-\frac{1}{h_{m}} \mathbf{R}-\mathbf{Q}_{\mathbf{2}} .
\end{aligned}
$$

$S^{\perp}$ is an right orthogonal complement of $S$.

Proof 1 Define the following Lyapunov-Krasovskii functional candidate:

$$
\begin{aligned}
V\left(x_{t}\right)= & x_{t}^{T}(0) P x_{t}(0)+\int_{-h(t)}^{0} x_{t}^{T}(\theta) Q_{1} x_{t}(\theta) d \theta \\
& +\int_{-h_{m}}^{0} x_{t}^{T}(\theta) Q_{2} x_{t}(\theta) d \theta \\
& +\int_{t-h_{m}}^{t} \int_{\theta}^{t} \dot{x}^{T}(s) R \dot{x}(s) d s d \theta
\end{aligned}
$$

Remark that since $P, Q_{1}, Q_{2}, R$ are positive definite, we can conclude that for some $\epsilon>0$, the Lyapunov-Krasovskii functional condition $V\left(x_{t}\right) \geq \epsilon\left\|x_{t}(0)\right\|$ is satisfied [3]. The derivative along the trajectories of (1) leads to

$$
\begin{aligned}
\dot{V}\left(x_{t}\right)= & 2 x^{T}(t) P \dot{x}(t)+x^{T}(t) Q_{1} x(t) \\
& -(1-\dot{h}(t)) x^{T}(t-h(t)) Q_{1} x(t-h(t)) \\
& +x^{T}(t) Q_{2} x(t)-x^{T}\left(t-h_{m}\right) Q_{2} x\left(t-h_{m}\right) \\
& +h_{m} \dot{x}^{T}(t) R \dot{x}(t)-\int_{t-h_{m}}^{t} \dot{x}^{T}(\theta) R \dot{x}(\theta) d \theta .
\end{aligned}
$$

As noted in [1]], the derivative of $\int_{t-h_{m}}^{t} \int_{\theta}^{t} \dot{x}^{T}(s) R \dot{x}(s) d s d \theta$ is often estimated as $h_{m} \dot{x}^{T}(t) R \dot{x}(t)-\int_{t-h(t)}^{t} \dot{x}^{T}(\theta) R \dot{x}(\theta) d \theta$ and the term $-\int_{t-h_{m}}^{t-h(t)} \dot{x}^{T}(\theta) R \dot{x}(\theta) d \theta$ 
is ignored, which may lead to considerable conservatism. Hence, the last term of (\$) can be separated in two parts:

$$
\begin{aligned}
-\int_{t-h_{m}}^{t} \dot{x}^{T}(\theta) R \dot{x}(\theta) d \theta= & -\int_{t-h_{m}}^{t-h(t)} \dot{x}^{T}(\theta) R \dot{x}(\theta) d \theta \\
& -\int_{t-h(t)}^{t} \dot{x}^{T}(\theta) R \dot{x}(\theta) d \theta .
\end{aligned}
$$

Using the Jensen's inequality [97, (9) can be bounded as follow:

$$
\begin{aligned}
& -\int_{t-h_{m}}^{t-h(t)} \dot{x}^{T}(\theta) R \dot{x}(\theta) d \theta-\int_{t-h(t)}^{t} \dot{x}^{T}(\theta) R \dot{x}(\theta) d \theta \\
& <-v^{T}(t) \frac{R}{h_{m}-h(t)} v(t)-w^{T}(t) \frac{R}{h(t)} w(t) \\
& <-v^{T}(t) \frac{R}{h_{m}} v(t)-w^{T}(t) \frac{R}{h_{m}} w(t)
\end{aligned}
$$

with

$$
\begin{aligned}
v(t) & =x(t-h(t))-x\left(t-h_{m}\right), \\
w(t) & =x(t)-x(t-h(t)) .
\end{aligned}
$$

Therefore, we get $\dot{V}\left(x_{t}\right)<\xi^{T}(t) \Gamma \xi(t)$ with $\Gamma$ defined as (6) and

$$
\xi(t)=\left[\begin{array}{c}
\dot{x}(t) \\
x(t) \\
x(t-h(t)) \\
x\left(t-h_{m}\right)
\end{array}\right] .
$$

Furthermore, using the extended variable $\xi(t)$, system (1) can be rewritten as $S \xi=0$ with $S$ defined as (5). The original system (1) is asymptotically stable if for all $\xi$ such that $S \xi=0$, the inequality $\xi^{T} \Gamma \xi<0$ holds. Using Finsler lemma [19], this is equivalent to $S^{\perp^{T}} \Gamma S^{\perp}<0$, where $S^{\perp}$ is a right orthogonal complement of $S$, which concludes the proof.

Note that Condition (何) can be rewritten as

$$
\begin{aligned}
& {\left[\begin{array}{ccc}
A^{T} P+P A+Q_{1}+Q_{2} & P A_{d} & 0 \\
A_{d}^{T} P & -(1-d) Q_{1} & 0 \\
0 & 0 & -Q_{2}
\end{array}\right]} \\
& \left.-\frac{1}{h_{m}}\left[\begin{array}{cc}
-1 & 0 \\
1 & -1 \\
0 & 1
\end{array}\right]\left[\begin{array}{cc}
R & 0 \\
0 & R
\end{array}\right]\left[\begin{array}{cc}
-1 & 0 \\
1 & -1 \\
0 & 1
\end{array}\right]\right]^{T} \\
& +h_{m}\left[\begin{array}{c}
A^{T} \\
A_{d}^{T} \\
0
\end{array}\right] R\left[\begin{array}{c}
A^{T} \\
A_{d}^{T} \\
0
\end{array}\right]^{T}<0 .
\end{aligned}
$$

Thus, according to this latter expression, we can conclude that if the LMI (11) is feasible for a given $h_{m}>0$, then it is feasible also for all delays less than the prescribed upperbound $h_{m}$.

Remark 1 Instead of using an orthogonal complement of $S$, Finsler lemma also states that condition $S^{\perp^{T}} \Gamma S^{\perp}<0$ is equivalent to the existence of some $X \in$ 
$\mathrm{R}^{4 \mathrm{n} \times \mathrm{n}}$ such that the $L M I \Gamma+X S+S^{T} X^{T}<0$ holds. Creating such additional variable $X$ is useless for the considered case: it only increases the number of variables and constraints in the LMI problem without reducing conservatism of the approach. But as demonstrated in [15], [0] and many others, such additional "slack variables" are of major interest for robust analysis purpose.

Remark 2 Note that delay-dependent results for fast varying delay (i.e. proving stability whatever the positive bound d) are a special case of the theorem 1. Fixing $Q_{1}=0$ renders the conditions independent on $d$ and therefore gives conditions for possibly fast varying delays.

\section{Main results}

\subsection{An augmented state for modelling the delayed systems}

As it has been noted, Theorem 1 is not a new result but a new formulation of existing equivalent results with fewer decision variables. Here, we aim at developing further the methodology used in the previous section to derive less conservative results. The key idea is that since the delay-dependent criterion proposed depends also on the derivative of the delay, we should highlight the relation between $\dot{h}(t)$ and states variables. One way is to consider an extended state $z=\left[\begin{array}{ll}x^{T} & \dot{x}^{T}\end{array}\right]^{T}$ as it has been proposed in [3] in a robustness context.

Differentiating the system (1), we get:

$$
\ddot{x}(t)=A \dot{x}(t)+(1-\dot{h}(t)) A_{d} \dot{x}(t-h(t)) .
$$

Introducing derivative of system (11) should provide more information on the system and hence improve results. Consider the artificially augmented system

$$
\left\{\begin{array}{l}
\dot{x}(t)=A x(t)+A_{d} x(t-h(t)) \\
\ddot{x}(t)=A \dot{x}(t)+(1-\dot{h}(t)) A_{d} \dot{x}(t-h(t))
\end{array}\right.
$$

with accordingly defined initial conditions. Introducing the augmented state

$$
z(t)=\left[\begin{array}{c}
x(t) \\
\dot{x}(t)
\end{array}\right]
$$

and specifying the relationship between the two components of $z(t)$ with the equality $\left[\begin{array}{ll}1 & 0\end{array}\right] \dot{z}(t)=\left[\begin{array}{ll}0 & 1\end{array}\right] z(t)$, we have the new augmented system

$$
E \dot{z}(t)=\bar{A} z(t)+\bar{A}_{d} z(t-h(t)),
$$

where

$$
\begin{gathered}
E=\left[\begin{array}{ll}
1 & 0 \\
0 & 1 \\
1 & 0
\end{array}\right], \quad \bar{A}=\left[\begin{array}{ll}
A & 0 \\
0 & A \\
0 & 1
\end{array}\right], \\
\bar{A}_{d}=\left[\begin{array}{cc}
A_{d} & 0 \\
0 & (1-\dot{h}(t)) A_{d} \\
0 & 0
\end{array}\right] .
\end{gathered}
$$

Finally, we obtain a descriptor linear time delay and time varying system, which may be more difficult to handle. Applying methodology developped in 
Section 2 to (15), the stability would be guaranteed only for a fixed $\dot{h}(t)$ since this term appears in $\bar{A}_{d}$. A common idea consists in embedding the time varying parameters $h$ and $\dot{h}$ into an uncertain set, described by a polytopic set and employing quadratic stability framework (see [2] and [9]).

Theorem 2 Define matrices $\mathcal{A}, \mathcal{B}$ and $\Theta_{2}$ as (25) and (20). Given scalars $h_{m}>0$ and $d \geq 0$, the linear system (A) is asymptotically stable for any timevarying delay $h(t)$ satisfying (国) and (可) if there exists $2 n \times 2 n$ matrices $P>0$, $Q_{j}>0, j=\{1,2\}$ and $R>0$ such that the following LMI holds for $i=\{1,2\}$ :

$$
\left[\begin{array}{cc}
\mathcal{A}^{(i)}-\frac{1}{h_{m}} \mathcal{B} & \Theta_{2}^{(i)^{T}} \mathbf{R} \\
\mathbf{R} \Theta_{2}^{(i)} & -\frac{1}{h_{m}} \mathbf{R}
\end{array}\right]<0
$$

where $\mathcal{A}^{(i)}\left(\Theta_{2}^{(i)}\right)$ for $i=1,2$ are the two vertices of $\mathcal{A}(\dot{h}) \in \mathrm{R}^{5 \mathrm{n} \times 5 \mathrm{n}}\left(\Theta_{2}(\dot{h}) \in\right.$ $\mathrm{R}^{2 \mathrm{n} \times 5 \mathrm{n}}$ respectively), replacing the term $\dot{h}(t)$ by $d_{i} . d_{i}, i=\{1,2\}$ corresponding to the bounds of $\dot{h}(t): d_{1}=d$ and $d_{2}=-d$.

Proof 2 We now consider the following Lyapunov-Krasovskii functional associated with the augmented state vector $z(t)$ :

$$
\begin{aligned}
V\left(z_{t}\right)= & z_{t}^{T}(0) P z_{t}(0)+\int_{-h(t)}^{0} z_{t}^{T}(\theta) Q_{1} z_{t}(\theta) d \theta \\
& +\int_{-h_{m}}^{0} z_{t}^{T}(\theta) Q_{2} z_{t}(\theta) d \theta \\
& +\int_{t-h_{m}}^{t} \int_{\theta}^{t} \dot{z}^{T}(s) R \dot{z}(s) d s d \theta .
\end{aligned}
$$

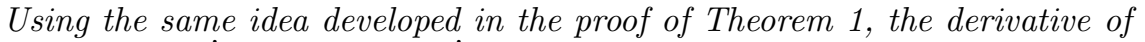
18) is such that $\dot{V}\left(z_{t}\right) \leq \psi(t)^{T} \Gamma(\dot{h}) \psi(t)$ where

$$
\begin{gathered}
\psi(t)=\left[\begin{array}{c}
\dot{z}(t) \\
z(t) \\
z(t-h(t)) \\
z\left(t-h_{m}\right)
\end{array}\right], \\
\Gamma(\dot{h})=\left[\begin{array}{cccc}
h_{m} R & P & 0 & 0 \\
P & T & \frac{1}{h_{m}} R & 0 \\
0 & \frac{1}{h_{m}} R & U & \frac{1}{h_{m}} R \\
0 & 0 & \frac{1}{h_{m}} R & V
\end{array}\right]
\end{gathered}
$$

with

$$
\begin{aligned}
T & =Q_{1}+Q_{2}-\frac{1}{h_{m}} R, \\
U & =-(1-\dot{h}(t)) Q_{1}-\frac{2}{h_{m}} R, \\
V & =-\frac{1}{h_{m}} R-Q_{2} .
\end{aligned}
$$

So, the system (10) is asymptotically stable if for all $\psi$ such that $S(\dot{h}) \psi=0$ with

$$
S(\dot{h})=\left[\begin{array}{llll}
-E & \bar{A} & \bar{A}_{d} & 0
\end{array}\right],
$$


the inequality $\psi(t)^{T} \Gamma(\dot{h}) \psi(t)<0$ holds. Using Finsler lemma [19], this is equivalent to

$$
S^{\perp^{T}}(\dot{h}) \Gamma(\dot{h}) S^{\perp}(\dot{h})<0
$$

where $S^{\perp}(\dot{h})$ is a right orthogonal complement of $S(\dot{h})$ given by

$$
S^{\perp}(\dot{h})=\left[\begin{array}{ccccc}
A & A_{d} & 0 & 0 & 0 \\
A A & A A_{d} & (1-\dot{h}) A_{d} & 0 & 0 \\
1 & 0 & 0 & 0 & 0 \\
A & A_{d} & 0 & 0 & 0 \\
0 & 1 & 0 & 0 & 0 \\
0 & 0 & 1 & 0 & 0 \\
0 & 0 & 0 & 1 & 0 \\
0 & 0 & 0 & 0 & 1
\end{array}\right] .
$$

Carrying out algebraic calculus of (22) with (20), condition (24) is derived:

$$
\mathcal{A}(\dot{h})-\frac{1}{h_{m}} \mathcal{B}+h_{m} \Theta_{2}^{T}(\dot{h}) R \Theta_{2}(\dot{h})<0
$$

where

$$
\begin{aligned}
\mathcal{A}(\dot{h}) & =\Theta_{1}^{T} P \Theta_{2}(\dot{h})+\Theta_{2}^{T}(\dot{h}) P \Theta_{1} \\
& +\Theta_{3}^{T}\left[\begin{array}{cc}
Q_{1} & 0 \\
0 & -(1-\dot{h}) Q_{1}
\end{array}\right] \Theta_{3} \\
& +\Theta_{4}^{T}\left[\begin{array}{cc}
Q_{2} & 0 \\
0 & -Q_{2}
\end{array}\right] \Theta_{4}, \\
\mathcal{B} & =\Theta_{5}^{T}\left[\begin{array}{cc}
R & 0 \\
0 & R
\end{array}\right] \Theta_{5} .
\end{aligned}
$$

and

$$
\begin{aligned}
\Theta_{1} & =\left[\begin{array}{ccccc}
1 & 0 & 0 & 0 & 0 \\
A & A_{d} & 0 & 0 & 0
\end{array}\right], \\
\Theta_{2}(\dot{h})= & {\left[\begin{array}{ccccc}
A & A_{d} & 0 & 0 & 0 \\
A^{2} & A A_{d} & (1-\dot{h}) A_{d} & 0 & 0
\end{array}\right], } \\
\Theta_{3} & =\left[\begin{array}{ccccc}
1 & 0 & 0 & 0 & 0 \\
A & A_{d} & 0 & 0 & 0 \\
0 & 1 & 0 & 0 & 0 \\
0 & 0 & 1 & 0 & 0
\end{array}\right] \\
\Theta_{4} & =\left[\begin{array}{ccccc}
1 & 0 & 0 & 0 & 0 \\
A & A_{d} & 0 & 0 & 0 \\
0 & 0 & 0 & 1 & 0 \\
0 & 0 & 0 & 0 & 1
\end{array}\right] \\
\Theta_{5} & =\left[\begin{array}{ccccc}
0 & 1 & 0 & -1 & 0 \\
0 & 0 & 1 & 0 & -1 \\
1 & -1 & 0 & 0 & 0 \\
\mathrm{~A} & A_{d} & -1 & 0 & 0
\end{array}\right]
\end{aligned}
$$
tion (24) is equivalent to

$$
\left[\begin{array}{cc}
\mathcal{A}(\dot{h})-\frac{1}{h_{m}} \mathcal{B} & \Theta_{2}^{T}(\dot{h}) \mathbf{R} \\
\mathbf{R} \Theta_{2}(\dot{h}) & -\frac{1}{h_{m}} \mathbf{R}
\end{array}\right]<0
$$


At this stage, assume that $\dot{h}(t)$ is not precisely known but varies between a lower and upper bound, $\dot{h}(t) \in\left[d_{1}, d_{2}\right]$. Since this uncertain parameter appears linearly in (2\%), the uncertain set can be described by a polytope 197. The vertices of this set can be calculated by setting the parameter to either lower or upper limit. The inequality (2y) can then be rewritten as follow:

$$
\left[\begin{array}{cc}
\sum_{i=1}^{2} \alpha_{i} \mathcal{A}^{(i)}-\frac{1}{h_{m}} \mathcal{B} & \sum_{i=1}^{2} \alpha_{i} \Theta_{2}^{(i)^{T}} \mathbf{R} \\
\mathbf{R} \sum_{i=1}^{2} \alpha_{i} \Theta_{2}^{(i)} & -\frac{1}{h_{m}} \mathbf{R}
\end{array}\right]<0
$$

where $\alpha_{i}(t) \in[0,1], \sum_{i=1}^{2} \alpha_{i}(t)=1$ and $\mathcal{A}^{(i)}\left(\Theta_{2}^{(i)}\right), i=1,2$ are the two vertices of the uncertain matrix $\mathcal{A}(\dot{h})\left(\Theta_{2}(\dot{h})\right.$ respectively) for $\dot{h}(t) \in\left[d_{1}, d_{2}\right]$. Considering the quadratic stability framework [1], condition (28) is equivalent to

$$
\left[\begin{array}{cc}
\mathcal{A}^{(i)}-\frac{1}{h_{m}} \mathcal{B} & \Theta_{2}^{(i)^{T}} \mathbf{R} \\
\mathbf{R} \Theta_{2}^{(i)} & -\frac{1}{h_{m}} \mathbf{R}
\end{array}\right]<0, \mathrm{i}=1,2 .
$$

Thus, the inequality (27) has to be verified only on its vertices (29). Finally, the asymptotic stability of system (15) is guaranteed if the two LMI (29) are feasible at the same time. For any initial conditions, the whole state $z(t)$ converges asymptotically to zero. Its components $x(t)$ converge as well. The original system (17) is asymptotically stable.

Remark 3 In the same way that in Section 6 for Theorem 1, if condition (24) holds for $h_{m}$ then it still holds for $h(t) \leq h_{m}$.

\subsection{A new Lyapunov functional}

The proposed new functional is based on the extension of a classical LyapunovKrasovskii functional (7). In order to take into account the variable $\ddot{x}(t)$, let introduce a new term for the Lyapunov-Krasovskii functional.

$$
\begin{aligned}
& V\left(z_{t}\right)=z_{t}^{T}(0) P z_{t}(0)+\int_{-h(t)}^{0} z_{t}^{T}(\theta) Q z_{t}(\theta) d \theta \\
& +\int_{t-h_{m}}^{t} \int_{\theta}^{t} \dot{z}^{T}(s) R \dot{z}(s) d s d \theta+\int_{t-h_{m}}^{t} \int_{s}^{t} \int_{u}^{t} \ddot{x}^{T}(\theta) W \ddot{x}(\theta) d \theta d u d s
\end{aligned}
$$

Then, we can propose the following result.

Theorem 3 Given scalars $h_{m}>0, d \geq 0$, the linear system (1) is asymptotically stable for any time-varying delay $h(t)$ satisfying (6) and (B) if there exists $2 n \times 2 n$ matrices $P>0, Q>0, R>0$, a $n \times n$ matrix $W>0$ and a matrix $X \in \mathrm{R}^{7 \mathrm{n} \times 4 \mathrm{n}}$ such that the following LMI holds for $i=\{1,2,3,4\}$ :

$$
\begin{aligned}
& {\left[\begin{array}{ccc}
\Gamma & \mathcal{E}^{T} \Theta_{3}^{(i)^{T}} \mathbf{R} & \mathcal{E}^{T} \Theta_{3}^{(i)^{T}} E_{2}^{T} \mathbf{W} \\
\mathbf{R} \Theta_{3}^{(i)} \mathcal{E} & -\frac{1}{h_{m}} \mathbf{R} & 0 \\
\mathbf{W} E_{2} \Theta_{3}^{(i)} \mathcal{E} & 0 & -\frac{2}{h_{m}^{2}} \mathbf{W}
\end{array}\right]<0} \\
& \Gamma=\mathcal{A}^{(i)}+\mathbf{X} S^{(i)}+S^{(i)^{T}} \mathbf{X}^{T}
\end{aligned}
$$

where $\mathcal{A}^{(i)}, \Theta_{3}^{(i)}$ and $S^{(i)}$ for $i=1,2,3,4$ are the vertices of matrices $\mathcal{A}(h, \dot{h}) \in$ $\mathrm{R}^{7 \mathrm{n} \times 7 \mathrm{n}}, \Theta_{3}(\dot{h}) \in \mathrm{R}^{2 \mathrm{n} \times 2 \mathrm{n}}$ and $S(h, \dot{h}) \in \mathrm{R}^{4 \mathrm{n} \times 7 \mathrm{n}}$ respectively, replacing the terms 
$h$ by 0 and $h_{m}$ and $\dot{h}(t)$ by $d$ and $-d . \mathcal{A}, S, \mathcal{E}$ and $\Theta_{3}$ are defined as (38) and (39) and (41).

Proof 3 First, let define the two matrices $E_{1}=\left[\begin{array}{ll}1_{n} & 0_{n}\end{array}\right]$ and $E_{2}=\left[\begin{array}{ll}0_{n} & 1_{n}\end{array}\right]$. Consider the Lyapunov-Krasovskii functional (30). Let us derive this quantity:

$$
\begin{aligned}
\dot{V} \leq & 2 \dot{z}^{T}(t) P z(t)+z^{T}(t) Q z(t) \\
& -(1-\dot{h}(t)) z^{T}(t-h(t)) Q z(t-h(t)) \\
& +h_{m} \dot{z}^{T}(t) Q R \dot{z}(t) \\
& -[z(t)-z(t-h(t))]^{T} \frac{R}{h(t)}[z(t)-z(t-h(t))] \\
& +\frac{h_{m}^{2}}{2} \dot{z}^{T} E_{2}^{T} W E_{2} \dot{z} \\
& -\left[h(t) E_{1} \dot{z}(t)-E_{1}(z(t)-z(t-h(t)))\right]^{T} \frac{W}{h^{2}(t) / 2} \\
& {\left[h(t) E_{1} \dot{z}(t)-E_{1}(z(t)-z(t-h(t)))\right] . }
\end{aligned}
$$

The last term of the inequality is not linear with respect to $h(t)$. Introducing the following signals

$$
\delta_{1}(t)=\frac{z(t)-\delta_{0}}{h(t)} \text { and } \delta_{2}(t)=\dot{z}(t)-\delta_{1} .
$$

allow to transform the right hand side of (32) into

$$
\begin{aligned}
\dot{V} \leq & 2 \dot{z}^{T}(t) P z(t)+z^{T}(t) Q z(t) \\
& -(1-\dot{h}(t)) z^{T}(t-h(t))^{T} Q z(t-h(t)) \\
& +h_{m} \dot{z}^{T}(t) R \dot{z}(t)-h(t) \delta_{1}^{T}(t) R \delta_{1}(t) \\
& +\frac{h_{m}^{2}}{2} \dot{z}^{T} E_{2}^{T} W E_{2} \dot{z}-2 \delta_{2}^{T}(t) E_{1}^{T} Q_{2} E_{1} \delta_{2}(t)
\end{aligned}
$$

Defining two extended vectors:

$$
\xi(t)=\left[\begin{array}{c}
z(t) \\
\dot{z}(t) \\
z(t-h(t)) \\
\delta_{1}(t) \\
\delta_{2}(t)
\end{array}\right] \text { and } \psi(t)=\left[\begin{array}{c}
x(t) \\
z(t-h(t)) \\
\delta_{1}(t) \\
\delta_{2}(t)
\end{array}\right]
$$

In equation (34) can be expressed as

$$
\dot{V} \leq \xi^{T}\left[\begin{array}{ccccc}
Q & P & 0 & 0 & 0 \\
P & T & 0 & 0 & 0 \\
0 & 0 & -(1-\dot{h}) Q & 0 & 0 \\
0 & 0 & 0 & -h R & 0 \\
0 & 0 & 0 & 0 & -2 E_{1}^{T} W E_{1}
\end{array}\right] \xi .
$$

with $T=h_{m} R+\frac{h_{m}^{2}}{2} E_{2}^{T} W E_{2}$. Then, specifying expressions of signals $\dot{x}$ and $\ddot{x}$ the following inequality is deduced.

$$
\dot{V} \leq \psi^{T}(t) N^{T} M N \psi(t)
$$


where $M$ is the matrix of the inequality (30) and

$$
N=\left[\begin{array}{cccc}
\Theta_{1} & 0_{2 n} & 0_{2 n} & 0_{2 n \times n} \\
\Theta_{2} & \Theta_{3}(\dot{h}) & 0_{2 n} & 0_{2 n \times n} \\
0_{2 n \times n} & 1_{2 n} & 0_{2} & 0_{2 n} \\
0_{2 n \times n} & 0_{2 n} & 1_{2} & 0_{2 n} \\
0_{2 n \times n} & 0_{2 n} & 0_{2} & 1_{2 n}
\end{array}\right]
$$

with

$$
\begin{aligned}
\Theta_{1} & =\left[\begin{array}{cc}
1 & 0 \\
A & A_{d}
\end{array}\right], \\
\Theta_{3}(\dot{h}) & =\left[\begin{array}{cc}
0 & \Theta_{2}=\left[\begin{array}{cc}
A & A_{d} \\
A^{2} & A A_{d}
\end{array}\right], \\
(1-\dot{h}) A_{d} & 0
\end{array}\right] .
\end{aligned}
$$

So, we get the inequality (37) under the constraint $S \psi=0$ with

$$
S(h, \dot{h})=\left[\begin{array}{ccccccc}
1 & -1 & 0 & -h 1 & 0 & 0 & 0 \\
A & A_{d} & -1 & 0 & -h 1 & 0 & 0 \\
A & A_{d} & 0 & -1 & 0 & -1 & 0 \\
A^{2} & A A_{d} & (1-\dot{h}) A_{d} & 0 & -1 & 0 & -1
\end{array}\right] .
$$

Using Finsler's lemma [19], equation (37) is equivalent to the following

$$
\begin{aligned}
\dot{V} \leq & \psi^{T}(t)\left[\mathcal{A}(h, \dot{h})+X S(h, \dot{h})+S^{T}(h, \dot{h}) X^{T}\right. \\
& \left.+\mathcal{E}^{T} \Theta_{3}^{T}(\dot{h}) T \Theta_{3}(\dot{h}) \mathcal{E}\right] \psi(t)
\end{aligned}
$$

with

$$
\begin{aligned}
& \mathcal{A}(h, \dot{h})=N^{T} M N-\mathcal{E}^{T} \Theta_{3}^{T}(\dot{h}) T \Theta_{3}(\dot{h}) \mathcal{E} \\
& \mathcal{E}=\left[\begin{array}{llll}
0_{2 \mathrm{n}} & 1_{2 \mathrm{n}} & 0_{2 \mathrm{n}} & 0_{\mathrm{n} \times 2 \mathrm{n}}
\end{array}\right] \\
& X \in \mathrm{R}^{7 \mathrm{n} \times 4 \mathrm{n}} \text { is a decision variable. }
\end{aligned}
$$

Then, applying twice the Schur's complement, expression (31) of Theorem 3 is obtained. Since $h$ and $\dot{h}$ appear linearly in (31) and using similar arguments as in the proof of Theorem [9, if the condition (31) is satisfied then the system (15) is asymptotically stable. As previously, since the whole state $z$ converges asymptotically to zero, its first component $x$ converges as well.

\section{Numerical example}

Consider the following system,

$$
\dot{x}(t)=\left[\begin{array}{cc}
-2 & 0 \\
0 & -0.9
\end{array}\right] x(t)+\left[\begin{array}{cc}
-1 & 0 \\
-1 & -1
\end{array}\right] x(t-h(t)) .
$$

For this academic example, many results were obtained in the literature. For various $d$, the maximal allowable delay, $h_{m}$, is computed. To demonstrate the effectiveness of our criterion, results are compared against those obtained in [4], [5], 20], [10], 11] and [12]. All these papers, except the last one, use the Lyapunov theory in order to derive some stability analysis criteria for time delay systems. In 12 , the stability problem is solved by a classical robust control 
Table 1: The maximal allowable delays $h_{m}$ for system (42)

\begin{tabular}{|c||c|c|c|c|c|c|c|c|c|c|}
\hline $\mathrm{d}$ & 0 & 0.1 & 0.2 & 0.5 & 0.8 & 1 & 1.1 & 1.2 & 1.3 & $\forall d$ \\
\hline \hline Fridman et al (2002) [4] & 4.472 & 3.604 & 3.033 & 2.008 & 1.364 & 0.999 & 0.999 & 0.999 & 0.999 & 0.999 \\
\hline Fridman et al (2006) [5] & 1.632 & 1.632 & 1.632 & 1.632 & 1.632 & 1.632 & 1.632 & 1.632 & 1.632 & 1.632 \\
\hline Wu et al (2004) [20] & 4.472 & 3.604 & 3.033 & 2.008 & 1.364 & - & - & - & - & - \\
\hline Kao et al (2005) [12] & 4.472 & 3.604 & 3.033 & 2.008 & 1.364 & 0.999 & - & - & - & - \\
\hline He et al (2007) [10] & 4.472 & 3.605 & 3.039 & 2.043 & 1.492 & 1.345 & 1.345 & 1.345 & 1.345 & 1.345 \\
\hline He et al (2007) [1] & 4.472 & 3.605 & 3.039 & 2.043 & 1.492 & 1.345 & 1.345 & 1.345 & 1.345 & 1.345 \\
\hline Theorem 1] & 4.472 & 3.605 & 3.039 & 2.043 & 1.590 & 1.345 & 1.345 & 1.345 & 1.345 & 1.345 \\
\hline Theorem 2 & 4.472 & 3.670 & 3.209 & 2.514 & 2.181 & 2.034 & 1.728 & 1.502 & 1.377 & - \\
\hline Theorem 3 & 5,120 & 4,081 & 3,448 & 2,528 & 2,152 & 1,991 & 1,575 & 1,271 & 1,108 & - \\
\hline
\end{tabular}

approach: the IQC framework. The results are shown in Table 1.

The numerical experiments show that Theorem 1 gives similar results to [10]. That seems logical since the same Lyapunov functional is used. Results for $d \geq 1$ and $\forall d$ are computed with Theorem 1 and choosing $Q_{1}=0$ in (6). [5 gives a rate-independent criterion which may be interesting (in certain cases as in example (42)) when $d$ is unknown. On the other hand, as no informations are taken into account about $\dot{h}(t)$, this could be conservative especially for small delay variations.

Then, considering the augmented system (15) composed by the original system (1) and its derivative, Theorem 2 improves the maximal allowable delays. Indeed, using the same Lyapunov-Krasovskii functional, conservatism is reduced thanks to the derivation of (11). As expected, this operation provides more information on the system and thus improves the stability analysis criterion.

Furthermore, Theorem 3 which consider an additional term (30) improves again the upperbound. This result suggests that the new proposed LyapunovKrasovskii functional (30) is suitable for time varying delay system stability analysis, reducing conservatism. However, in example 42 for $|\dot{h}| \geq 0.8$, Theorem 2 provides slightly better results than Theorem 3 . Nevertheless, this difference could be compensate by adding to the functional (30) the term $\int_{t-h_{m}}^{t} z^{T} Q_{2} z$ and applying the separation of the integral in the third term as (9).

\section{CONCLUSION}

In this paper, the problem of the delay dependent stability analysis of a time varying delay system has been studied by means of a new Lyapunov-Krasovskii functional. The first criterion is based on an existing Lyapunov-Krasovskii functional [10] (see Theorem 11). Based on this first result, and using an augmented state, new types of Lyapunov-Krasovskii functional are introduced which emphasizes the relation between $\dot{h}$ and signals $\dot{x}$ and $\ddot{x}$. The resulting criteria are then expressed in terms of a convex optimization problem with LMI constraints, allowing for the use of efficient solvers. Finally, a numerical example shows that these methods reduced conservatism and improved the maximal allowable delay. 


\section{References}

[1] P.-A. Bliman. Lyapunov equation for the stability of linear delay systems of retarded and neutral type. IEEE Trans. on Automat. Control, 47:327-335, February 2002.

[2] S. Boyd, L. El Ghaoui, E. Feron, and V. Balakrishnan. Linear Matrix Inequalities in System and Control Theory. SIAM, Philadelphia, USA, 1994. in Studies in Applied Mathematics, vol.15.

[3] Y. Ebihara, D. Peaucelle, D. Arzelier, and T. Hagiwara. Robust performance analysis of linear time-invariant uncertain systems by taking higherorder time-derivatives of the states. In $44^{\text {th }}$ IEEE Conference on Decision and Control and the European Control Conference, Seville, Spain, December 2005 .

[4] E. Fridman and U Shaked. An improved stabilization method for linear time-delay systems. IEEE Trans. on Automat. Control, 47:1931-1937, November 2002.

[5] E. Fridman and U Shaked. Input-output approach to stability and $l_{2}$-gain analysis of systems with time-varying delays. Systems \& Control Letters, 55:1041-1053, September 2006.

[6] F. Gouaisbaut and D. Peaucelle. Delay-dependent robust stability of time delay systems. In $5^{\text {th }}$ IFAC Symposium on Robust Control Design (ROCOND'06), Toulouse, France, July 2006.

[7] F. Gouaisbaut and D. Peaucelle. Delay-dependent stability analysis of linear time delay systems. In IFAC Workshop on Time Delay System (TDS'06), Aquila, Italy, July 2006.

[8] F. Gouaisbaut and D. Peaucelle. A note on stability of time delay systems. In $5^{\text {th }}$ IFAC Symposium on Robust Control Design (ROCOND'06), Toulouse, France, July 2006.

[9] K. Gu, V. L. Kharitonov, and J. Chen. Stability of Time-Delay Systems. Birkhäuser Boston, 2003. Control engineering.

[10] Y. He, Q. G. Wang, C. Lin, and M. Wu. Delay-range-dependent stability for systems with time-varying delay. Automatica, 43:371-376, 2007.

[11] Y. He, Q. G. Wang, L. Xie, and C. Lin. Further improvement of freeweighting matrices technique for systems with time-varying delay. IEEE Trans. on Automat. Control, 52:293-299, February 2007.

[12] C. Y. Kao and A. Rantzer. Robust stability analysis of linear systems with time-varying delays. In $16^{\text {th }}$ IFAC World Congress, Prague, Czech Republic, 2005.

[13] V. B. Kolmanovskii and J. P. Richard. Stability of some linear systems with delays. IEEE Trans. on Automat. Control, 44:984-989, May 1999. 
[14] S.I. Niculescu. Delay Effects on Stability. A Robust Control Approach, volume 269 of Lecture Notes in Control and Information Sciences. SpringerVerlag, Heildelberg, 2001.

[15] D. Peaucelle and F. Gouaisbaut. Discussion on parameter-dependent lyapunov functions approach to stability analysis and design for uncertain systems with time-varying delay. European J. of Control, 11(1):69-70, 2005.

[16] Dimitri Peaucelle, Denis Arzelier, Didier Henrion, and Frédéric Gouaisbaut. Quadratic separation for feedback connection of an uncertain matrix and an implicit linear transformation. Automatica, 43(5):795-804, 2007.

[17] J.-P. Richard. Time-delay systems: an overview of some recent advances and open problems. Automatica, 39:1667-1694, October 2003.

[18] Rifat Sipahi and Nejat Olgac. Stability robustness of retarded lti systems with single delay and exhaustive determination of their imaginary spectra. SIAM J. Control Optim., 45(5):1680-1696, 2006.

[19] R. Skelton, T. Iwazaki, and K. Grigoriadis. A unified algebric approach to linear control design. Taylor and Francis series in systems and control, 1998.

[20] M. Wu, Y. He, J. H. She, and G. P. Liu. Delay-dependent criteria for robust stability of time-varying delay systems. Automatica, 40:1435-1439, 2004.

[21] J. Zhang, C. R. Knopse, and P. Tsiotras. Stability of time-delay systems: Equivalence between Lyapunov and scaled small-gain conditions. IEEE Trans. on Automat. Control, 46(3):482-486, March 2001.

[22] J. Zhang, C. R. Knospe, and P. Tsiotras. Toward less conservative stability analysis of time delay systems. In Proc. 38th IEEE CDC'99, pages 20172022, Phoenix, Arizona, December 1999. 